\title{
Peptide biosensors for anticancer drugs: design in silico to work in denaturizing environment.
}

Filomena Guida ${ }^{\mathrm{a}}$, Anna Battisti ${ }^{\mathrm{b}}$, Ivan Gladich ${ }^{\mathrm{b}}$, Mauro Buzzo $^{\mathrm{a}, \mathrm{c}}$, Elena Marangon ${ }^{\mathrm{c}}$, Luciana Giodini $^{\mathrm{c}}$, Giuseppe Toffoli ${ }^{\mathrm{c}, *}$, Alessandro Laio $^{\mathrm{b}, *}$, Federico Berti $^{\mathrm{a}, *}$

${ }^{\mathrm{a}}$ :Dipartimento di Scienze Chimiche e Farmaceutiche, Università degli Studi di Trieste, Italy (filomena.guida@dia.units.it, fberti@units.it)

${ }^{\mathrm{b}}$ :International School for Advanced Studies (SISSA), Via Bonomea 265, I-34136 Trieste, Italy (abattisti@sissa.it,igladich@sissa.it, laio@sissa.it)

${ }^{\mathrm{c}}$ :CRO National Cancer Institute Experimental and Clinical Pharmacology Division, Department of Translational Research, Aviano, Italy (mbuzzo@cro.it, emarangon@cro.it, lgiodini@cro.it, gtoffoli@cro.it)

*Corresponding authors: Giuseppe Toffoli, Centro di Riferimento Oncologico, IRCCS, Via Franco Gallini 2, 33081 Aviano, Italy, +39 0434 659235, gtoffoli@cro.it; Alessandro Laio, Scuola Internazionale Superiore di Studi Avanzati, via Bonomea 265, 34136 Trieste, Italy +39 0403787426 , laio@sissa.it; Federico Berti, Dipartimento di Scienze Chimiche e Farmaceutiche, via Giorgieri 1, 34128 Trieste, Italy, +39040 5583920, fberti@units.it.

ABSTRACT: One of the main targets in current clinical oncology is the development of a cheap device capable of monitoring in real-time the concentration of a drug in the blood of a patient. This would allow fine-tuning the dosage according to the patient's metabolism, a key condition to reduce side effects. By using surface plasmon resonance and fluorescence spectroscopy we here show that short peptides designed in silico by a recently developed algorithm are capable of binding the anticancer 
drug irinotecan (CPT-11) with micromolar affinity. Importantly, the recognition takes place in the denaturating solution used in standard therapeutic drug monitoring to detach the drug from the proteins that are present in human plasma, and some of the peptides are capable of distinguishing CPT-11 from its metabolite SN-38. These results suggest that the in silico design of small artificial peptides is now a viable route for designing sensing units, opening a wide range of applications in diagnostic and clinical areas.

Keywords: peptides; drug monitoring; in silico design; irinotecan.

\section{Introduction}

An optimal therapeutic protocol for cancer should ensure the best response with minimal toxicity. However, achieving this goal is far from simple, since several factors may affect the drug concentration in the blood of a patient under treatment. These include, among the others, the gender, the age, the health status and pharmacogenomics variations in genes encoding enzymes involved in drug absorption and metabolism (Ma and Lu 2011). Therapeutic Drug Monitoring (TDM) aims at measuring directly the concentration of the drug in the blood of the patient. This allows, at least in principle, personalizing the treatment by adjusting the dosage and reducing side effects (Walko and McLeod 2014; De Jong et al. 2005; Alnaim 2007). It is particularly useful for monitoring drugs employed in long-term treatments or drugs that have a narrow therapeutic range. Drugs suitable for TDM are characterized by a known dosedependent response, a significant inter-individual pharmacokinetic variability, a narrow therapeutic window exposure-response relationship, and anticancer drugs meet these criteria (Paci et al. 2014). TDM involves measuring drug concentrations in plasma, serum or blood at designed time intervals, ideally in continuum. In the clinical care it is a routine practice only for few drugs. It represents a valuable guidance for antibiotics, immunosuppressives, antiepileptics, while for anticancer drugs is still uncommon (Gao et al. 2012; Widmer et al. 2014). Currently, the main technologies applied in the TDM 
of anticancer drugs are based on the extraction of the drug from blood or plasma samples, followed by HPLC or LC-MS (Marangon et al. 2014; Van Erp et al 2014) analysis. However, the HPLC instrumentation is expensive and generally available only to laboratories of hospitals and research centers. Developing a point of care device, that can be used directly at the patient bed or by the patient, with simple sample preparation, high robustness, sensitivity and good selectivity is still an open technological challenge. Such a device should ideally be based on a biosensor equipped with a stable and cheap sensing elements. A possible option is using an aptamer changing its conformation upon binding, as reported and tested on animal models (Ferguson et al. 2014).

Short peptides offer a viable alternative to aptamers for designing sensing elements for TDM, since they can be easily synthesized in large quantities, are cost-effective and bio-stable. Despite of these potentialities, their use in real-world application is still rather limited. Indeed, short peptides cannot be designed by exploiting a natural selection mechanism, like is done for antibodies. Designing the peptides in silico is also highly non trivial, due to their high flexibility and the large conformational changes which can be triggered by a single mutation. A further difficulty arises in the specific field of TDM: protein precipitation, the most common sample preparation procedure, in TDM is performed by adding to the patient sample a significant fraction of denaturant in order to detach the drug from the proteins to which it is bound (typically albumin). Since the binding affinity can change significantly in a denaturizing environment, the design cannot be carried on using as templates peptide fragments binding the target in water solutions.

In order to overcome the above-mentioned difficulties, we have recently developed a computational protocol providing a route to design short peptides with high affinity and selectivity for organic molecules in a solvent specific environment (Gladich et al. 2015). In short, the approach is based on a stochastic search in the peptide sequence space driven by a series of single point mutations. At each mutation step, a conformational sampling is performed by molecular dynamics simulation in explicit 
solvent environment and the mutation is accepted or rejected according to a Monte Carlo scheme targeted at improving the estimated stability of the complex. Modeling the solvent explicitly allows taking into account the non-trivial effect of the denaturant on the strength of the contacts stabilizing the drug-peptide complex. Recently, this computational scheme allowed the design of peptides capable to recognize chlorogenic acid by electrochemical detection in food samples (Del Carlo et al. 2016).

In this paper we analyzed theoretically and experimentally the peptides designed by the above computational approach and we give the prove of their capability to bind CPT-11 (Scheme 1) in a denaturant solution, namely in the conditions commonly used for TDM, showing evidence that short peptides designed in silico can be used as sensing elements for TDM of anticancer drugs. These peptides are, indeed, by construction solvent specific, i. e., capable to recognize the target in the solvent in which they have been designed. This calls for applications in devices in which the peptide-based sensor is required to work in denaturants or under extreme conditions of $\mathrm{pH}$ and/or salt concentration.

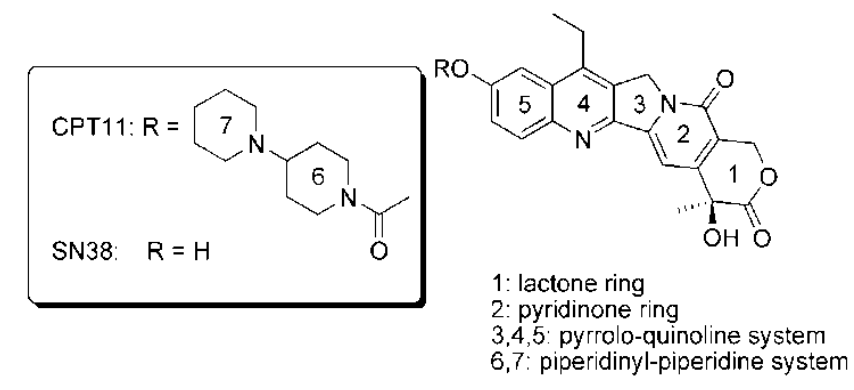

\section{Scheme 1}

\section{Materials and methods}

Design of peptide sequences: we used the procedure previously developed and described in detail in Gladich et al. 2011 in order to design short cyclic peptides with high binding affinity toward CPT-11. The search of the optimal sequence starts considering the target molecule inserted in a cyclic peptide of 12 VAL closed at the end by a disulfide bridge between two cysteines. We then randomly selected one amino acid from the peptide and replaced it with a different one, while the two terminal cysteines connected with a disulfide bridge are excluded to preserve the cyclic geometry of the peptide. In this 
way, the new peptide sequence at the step $\mathrm{i}+1, \mathrm{SEQ}_{\mathrm{i}+1}$, differs from the sequence at the previous step, $\mathrm{SEQ}_{i}$, for only one amino acid at the time. The stability of the complex after each mutation step were estimated by the mean value $E_{i}$ of Vina21 (Trott et al. 2010) scoring function calculated on the molecular dynamics (MD) trajectory. The new peptide sequence $\mathrm{SEQ}_{\mathrm{i}+1}$ is accepted or rejected according to the Metropolis criterion, with a probability $\min \left(1, \exp \left[\left(\mathrm{E}_{\mathrm{i}+1}-\mathrm{E}_{\mathrm{i}}\right) / \mathrm{T}_{\mathrm{e}}\right]\right)$ controlling the acceptance rate by an efficacious temperature, $\mathrm{T}_{\mathrm{e}}$. In the case of acceptance, a new mutation is attempted on the new structure, otherwise on the previous one. The mutation cycle was iterated until the estimated binding affinity reached a plateau (see Figure 1). In order to enhance the exploration of the sequence space, five simultaneous and independent mutation cycles at five efficacious temperatures $T_{e}$ $=0.2,0.4,0.6,0.8$, and $1.0 \mathrm{kcal} / \mathrm{mol}$ were performed, attempting to swap two replicas, $\mathrm{r}$ and $\mathrm{r}^{\prime}$, at the end of each mutation step. A swap between the states of replica $r$ at $T_{r},\left(S E Q_{r}, E_{r}\right)$ and the state of replica $\mathrm{r}^{\prime}$ at $\operatorname{Tr}^{\prime},\left(\mathrm{SEQ}_{\mathrm{r}^{\prime}}, \mathrm{E}_{\mathrm{r}^{\prime}}\right)$ was accepted with probability $\min \left(1, \exp \left[\left(\mathrm{E}_{\mathrm{r}}-\mathrm{E}_{\mathrm{r}^{\prime}}\right)\left(1 / \mathrm{T}_{\mathrm{r}}-1 / \mathrm{T}_{\mathrm{r}^{\prime}}\right)\right]\right)$.

This mutation cycle was iterated until the estimated binding affinity reached a plateau, visible in Figure 1. A set of different peptide sequences was selected from the outcome of the design cycle among those with higher binding affinity and with, at least, three amino acid difference in the sequences. In supplementary data we report an example of the design output with a table of the first 60 peptides with higher affinity toward CPT-11 in MeOH. Within this set we further selected between 5 to 10 sequences for a theoretical validation of their thermal stability, performing long MD trajectories at $300 \mathrm{~K}$. To test the stability of the complex we can choose the same setup used in the design, or a different environment. For example, we can change the solvent. In the present work the peptides 1-3 were designed in vacuum and tested in water and methanol on a $200 \mathrm{~ns}$ NPT simulations at $300 \mathrm{~K}$. Peptides 4-5 were designed specifically in methanol and tested in water and methanol. Peptides 1-5 in Table 1 were chosen for experimental validation among those that showed higher thermal stability and affinity over the course of 200 ns MD. 


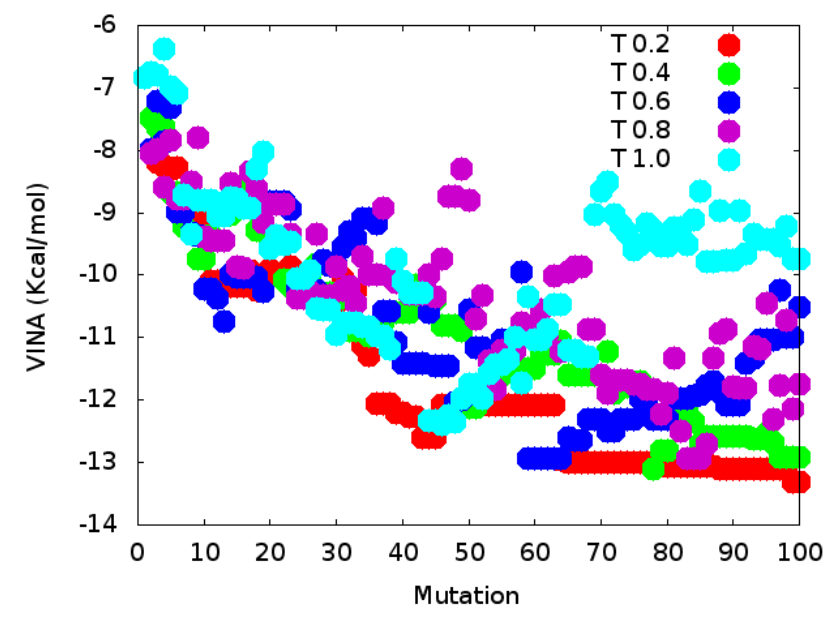

a

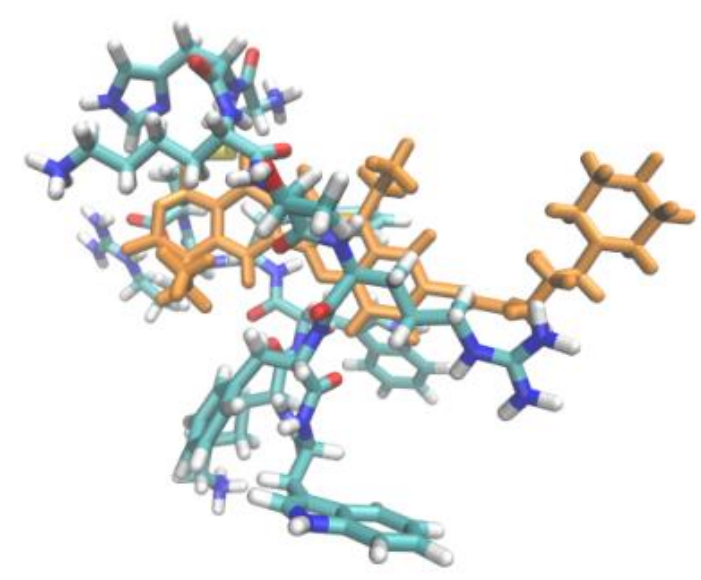

b

Figure 1

The MD runs were performed using GROMACS molecular packages (Hess et al. 2008). At each mutation step, we performed 500000 steps of steepest descent energy minimization in explicit solvent, followed by 50 ps NVT equilibration and afterwards by $5 \mathrm{~ns}$ constant temperature and pressure (NPT) run. During the NVT and NPT runs the temperature was kept at $300 \mathrm{~K}$. The peptide and solvent molecules were modelled by the AMBER99SB force field (Hornak et al. 2006), while GAFF force field was used for CPT-11. The two metabolites CPT-11 and SN-38 that we considered in this work are stable at $\mathrm{pH}=4.5$ and in this condition they are active as anticancer drugs. In the $4.5-7 \mathrm{pH}$ range, among the tetratable residues (Lys, Arg, Asp, Glu, His), only His can change the protonation state. Moreover, the effect of the $\mathrm{pH}$ on the protonation state of His in methanol is known only qualitatively. We therefore performed the design considering the neutral form of His. For a few selected configurations and sequences, we then computed the binding affinity using a protonated His; the difference on the VINA binding affinity is typically of the order of $1 \mathrm{kcal} / \mathrm{mol}$, significantly smaller than the statistical error. Therefore, in this specific case the effect of the change of the protonation of the amino acids can be neglected. Similarly, we did not take into account the effect on the binding affinity 
of the ionic strength, as our aim was to obtain peptides capable to bind their targets in an organic environment.

Design for experiments: to carry on some of the experiments it is necessary to immobilize the peptides on a surface, for example on the SPR chip. To this aim we added a short additional chain of amino acids at the amino terminus of the designed peptides, to act as a linker between the cyclic peptide and the surface. We have chosen for the linker the sequence LysGlyGlyGly and on a few cases we tested via molecular dynamic simulation that this linker do not affect the ligand-peptide binding affinity. Furthermore with molecular dynamic simulation we analyzed the effect of the presence of a surface on the structure of the complex: we modeled a $\mathrm{SiO} 2$, using the parameters as defined in the DREIDING force field. During the NVT dynamics $(\mathrm{T}=300 \mathrm{~K})$ kept the terminal amino group of Lys at a distance of 3.5 A from the surface. During the simulation the CPT11-peptide complex remains far from the surface, and we did not observe significant changes in the ligand-receptor interactions. Consistently with these theoretical results, we will show in the following that the binding affinity estimated by SPR and by florescence spectroscopy is very similar.

Surface Plasmon Resonance: the binding affinity was evaluated by Surface Plasmon Resonance using a BIAcore X100 instrument (GE Healthcare Europe GmbH). The peptides were immobilized on the CM5 sensor chip using a standard amine coupling in $10 \mathrm{mM}$ sodium acetate buffer (pH 4.5). As a reference surface, "flow cell 1" was treated with ethanolamine.

The binding was revealed by increasing CPT-11 concentrations in continuous flow; eight different concentrations of CPT-11 (from $0.5 \mu \mathrm{M}$ to $200 \mu \mathrm{M}$ ) were injected over each surface using $10 \mathrm{mM}$ sodium acetate buffer $\mathrm{pH} 4.5$ as running buffer and a flow rate of $30 \mu \mathrm{l} / \mathrm{min}$ for 180 sec. The dissociation step was performed using the same buffer and the same flow rate for 300 sec. Non-specific binding was eliminated subtracting the signal obtained from the reference flow cell. The final binding 
sensorgrams were analysed using the BIAevaluation software and by fitting the curves using the Steady State Affinity model.

Fluorescence spectroscopy: fluorescence spectra were recorded on a CARY Eclipse (Varian) spectrofluorimeter at $25{ }^{\circ} \mathrm{C}$. Concentrated mother solutions of peptides 1-3 were prepared in water, while peptides 4 and 5 were dissolved in methanol. The concentration of CPT-11 was fixed at 1 or 0.5 $\mu \mathrm{M}$ in $400 \mu \mathrm{L}$ of $\mathrm{MeOH}$. Peptide concentration was increased from $1 \mathrm{nM}$ to $100 \mu \mathrm{M}$ adding $2 \mu \mathrm{L}$ of the mother solutions for each concentration. The measurements were carried out in a $5 \mathrm{~mm}$ optical path cell. The excitation and emission wavelengths were respectively set at $220-430 \mathrm{~nm}$ or $267-425 \mathrm{~nm}$ in water and $\mathrm{MeOH}$. The excitation and emission slits were set at 5 and $10 \mathrm{~nm}$ respectively. All the experiments were repeated three times.

The quenching data obtained from the titrations were analysed according to the Stern-Volmer equation: $\mathrm{F}_{0} / \mathrm{F}=1+\mathrm{K}_{\mathrm{q}} \tau_{0}[\mathrm{Q}]=1+\mathrm{K}_{\mathrm{sv}}[\mathrm{Q}]$

Where $F_{0}$ and $F$ are the emission intensities before and after the addition of the quencher, respectively, $\mathrm{K}_{\mathrm{q}}$ is the bimolecular quenching kinetic constant, $\tau_{0}$ is the fluorescence decay time of the fluorophore (about 3.5 ns for CPT-11,) (Dey et al. 1997), $\mathrm{K}_{\mathrm{sv}}$ is the Stern-Volmer quenching constant and [Q] is the quencher concentration (peptide) in mol/L. At $1 \mu \mathrm{M} \mathrm{CPT-11,} \mathrm{the} \mathrm{Stern-Volmer} \mathrm{plots} \mathrm{were} \mathrm{linear} \mathrm{in} \mathrm{a} 0$ $100 \mu \mathrm{M}$ quencher peptides range, and the $\mathrm{K}_{\mathrm{sv}}$ values were determined by linear regressions; this allowed to estimate $\mathrm{K}_{\mathrm{q}}$ (as $\mathrm{K}_{\mathrm{sv}} / \tau_{0}$ ). The dissociation constant of the CPT-11-peptides complexes and the stoichiometry of their formation were then evaluated by a Hill analysis:

$\log (\Theta / 1-\Theta)=n \log [\mathrm{Q}]-\log \mathrm{K}_{\mathrm{D}}$

where $\Theta$ was calculated as $1-\mathrm{F} / \mathrm{F}_{0}$ and represents the fraction of free binding sites, [Q] is the quencher concentration in mol/L, $\mathrm{n}$ is the number of binding sites, and $\mathrm{K}_{\mathrm{D}}$ is the dissociation constant. 


\section{Results and discussion}

We designed 16-residue long peptides following the procedure described in Gladich et al. 2015. In order to reduce the entropic penalty in the unbound state, we designed cyclic peptides (Hong et al. 2012) keeping a disulfide bridge between the first and the last cysteine residues of the ring. Additional details on the computational procedure are reported in the Materials and methods section. In order to demonstrate the advantage of performing the design in explicit solvent, we (a) first considered peptides designed in vacuum with no explicit solvent and setting a simple cut-off on the non-bonded interaction and then (b) peptides designed in methanol, one of the solvent used in TDM to detach the drug from the protein.

\section{Binding affinity for peptides designed in vacuum}

The binding affinity in water solution of the three cyclic peptides designed in vacuo (Table 1) was first estimated experimentally by Surface Plasmon Resonance (SPR), then by fluorescence spectroscopy. SPR was our method of choice to obtain rapid and accurate determinations of the binding affinity in water environment. The peptides were immobilized on the sensor chip surface, and their N-termini were linked to the carboxylic groups of the dextran matrix on the sensor chip by standard amine coupling. After immobilization, CPT-11 was injected in continuous flow over the sensor surface at increasing concentrations. Binding analyses were performed in acidic running buffer $(10 \mathrm{mM}$ sodium acetate, $\mathrm{pH}$ 4.5) to protect the lactone ring of CPT-11 from opening, which occurs at neutral/basic $\mathrm{pH}$ (Fassberg et al. 1992). The experimental sensorgrams (Figure 2A) indicate that all three peptides have a good binding capacity towards CPT-11, showing the response units (RU) of binding curves in the sensorgrams increasing with CPT-11 concentration. Decreasing the concentration of the analyte down to $50 \mathrm{nM}$, the peptide maintains a detectable binding capacity, and the sensor gives a clean and reproducible signal. 
For all the three peptides, the association and dissociation kinetics of the peptide-CPT-11 complexes are very fast (see Table 1). This does not allow obtaining binding parameters from a kinetic analysis of the SPR data. However, using the steady-state values of RU, it was possible to calculate the dissociation constants $\left(\mathrm{K}_{\mathrm{D}}\right)$ from equibrium data with the BIAevaluation software. The affinity turned out to be 64 $\mu \mathrm{M}$ for peptide 1 (Table 1). Similar values of 63 and $62 \mu \mathrm{M}$ were obtained for peptides 2 and $\mathbf{3}$ respectively.
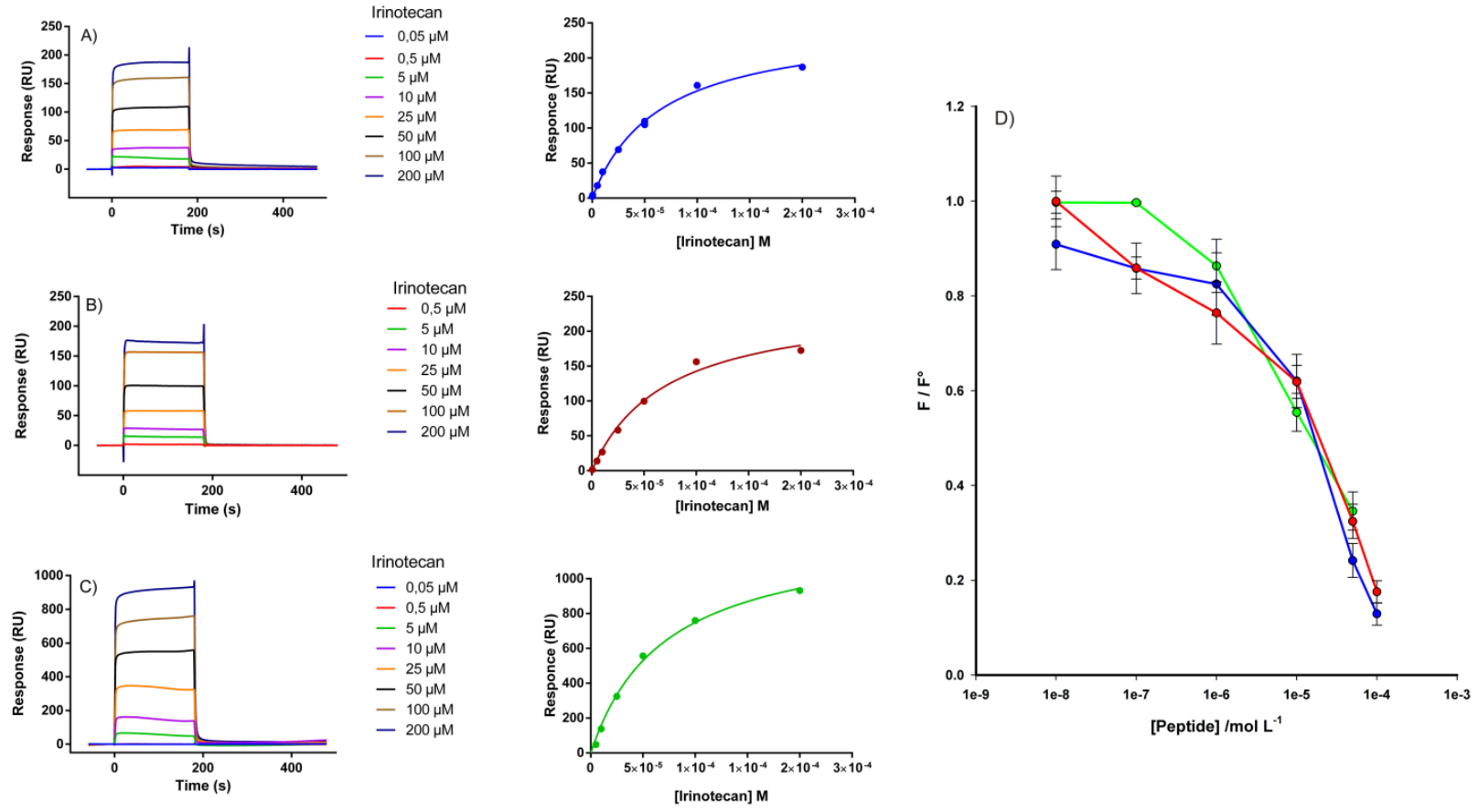

Figure 2

The binding activity of the three peptides designed in vacuo was also evaluated using fluorescence spectroscopy. This technique allows measuring the binding affinity both in water solution and in watermethanol mixtures, which are not compatible with the Biacore sensor. Therefore, it allows measuring the affinity also in the conditions that are relevant for TDM, and will be exploited for peptides specifically designed for this aim. As the peptides are poorly fluorescent, but they show a good ability to quench the fluorescence of CPT-11, we have designed a reversed experiment to obtain the binding 
affinity. The interaction was therefore studied monitoring the quenching of fluorescence of CPT-11, whose concentration was kept constant, upon increasing peptide concentrations. A control experiment was carried out on peptide $\mathbf{1}$, and in this case the recognition event was studied also by monitoring the tryptophan emission of the peptide $(280 / 340 \mathrm{~nm})$. Following CPT-11 fluorescence, the excitation and emission wavelengths were respectively set at 220 and $430 \mathrm{~nm}$. The experiments were carried out in both water and methanol. During the titrations, CPT-11 fluorescence quenching was observed increasing the peptide concentration in all cases (Figure 2D and 3A).

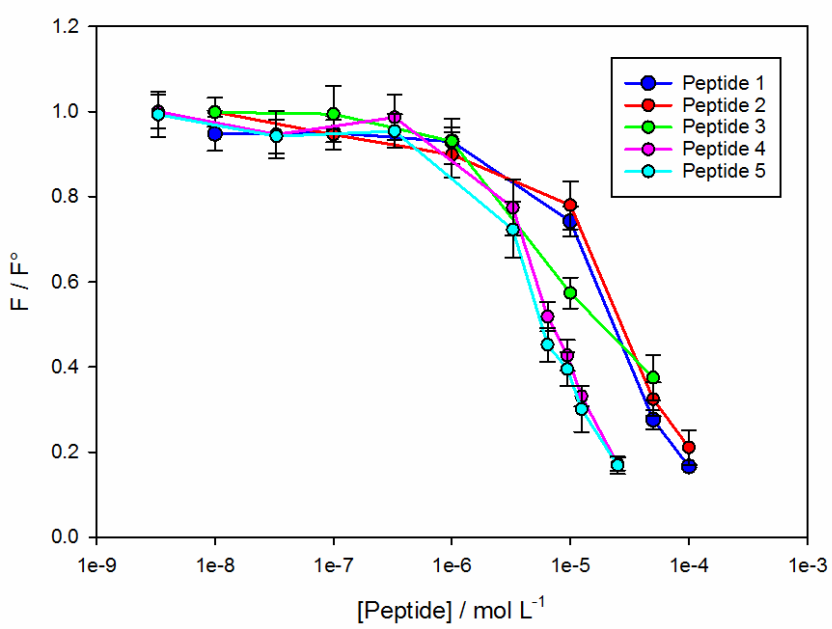

$\mathbf{a}$

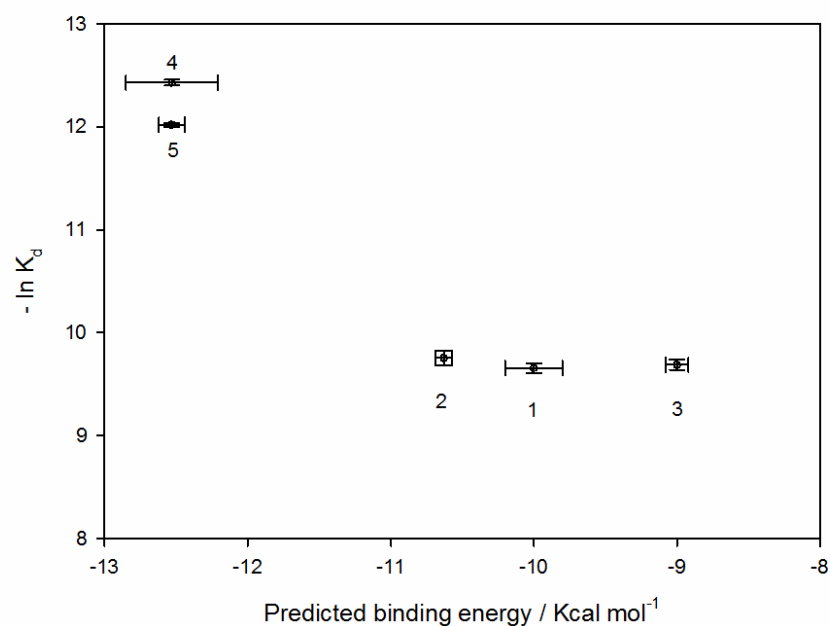

b

\section{Figure 3}

The quenching data were analyzed according to the Stern-Volmer equation (see materials and methods). In all cases the quenching constants were well over $10^{12} \mathrm{Lmol}^{-1} \mathrm{~s}^{-1}$, the limit value for collisional quenching. Having thus established that a static quenching phenomenon occurs, we have evaluated the dissociation constant of the CPT-11-peptides complexes and the stoichiometry of their formation by a Hill analysis of the fluorescence data (see methods). By this analysis, the peptides $\mathbf{1}, \mathbf{2}$ and $\mathbf{3}$ gave a $\mathrm{K}_{\mathrm{D}}$ of 23, 20 and $26 \mu \mathrm{M}$ in water and 41,53,56 $\mu \mathrm{M}$ in methanol respectively (Table 1). The affinity of all three peptides in aqueous solution, measured by fluorimetry, is thus consistent with the one measured by SPR. On moving from the water environment to methanol, the affinity decreases by a factor 3, bringing 
the $\mathrm{K}_{\mathrm{D}}$ in the medium micromolar range. The Hill number turned out to be 1 in methanol, indicating a 1:1 binding between peptide and CPT-11, while it is interesting to note an $n<1$ in aqueous solution for all three peptides. An explanation may be that, in aqueous solution, peptide self-aggregation occurs at high concentration, and, in this case, a fraction of binding sites may be hidden leading to the observed apparent negative cooperativity. The decrease of polarity in methanol might reduce the tendency to peptide - peptide hydrophobic aggregation thus making available the whole of the binding sites.

The experimental values of the binding affinity are in good agreement with those obtained by molecular dynamics simulation. For each of the three peptides designed in vacuum, we generated trajectories of the complex CPT-11-peptide in methanol, and for peptide 1 also in water (see Figure S1 in supplementary materials). In all the cases, the peptide-drug complexes are stable over all simulation times with an estimated binding affinity of about $-10 \mathrm{kcal} / \mathrm{mol}$ (see Figure S2).

\section{Binding affinity of the peptides designed in methanol}

We then considered cyclic peptides specifically designed in methanol, by taking explicitly into account the effect of the solvent interactions by an atomistic description. From this design, we selected two peptides, 4 and 5 hereafter (Table 2), whose theoretical binding affinity to CPT-11 in methanol is -12.5 $\mathrm{kcal} / \mathrm{mol}$. For these peptides, the experimental tests were carried out by fluorescence spectroscopy only, since the SPR sensor cannot be used in methanol, and peptides $\mathbf{4}$ and $\mathbf{5}$ are not soluble in water.

The fluorescence quenching experiment showed that a gain in affinity occurs, and the whole titration curve of fluorescence emission is shifted down by one order of magnitude (Figure 3). In order to evaluate the dissociation constants of peptides $\mathbf{4}$ and $\mathbf{5}$, it was necessary reducing the concentration of CPT-11 to $0.5 \mu \mathrm{M}$, In this manner, we obtained 4.1 and $6.2 \mu \mathrm{M} \mathrm{K} \mathrm{K}_{\mathrm{d}}$ s respectively (Table 2). The binding stoichiometry is 1:1 by Hill analysis in both cases. Theoretical and experimental binding affinities in methanol for all the five peptides analysed in this work are in fair agreement, as shown in Figure 3 B. 
This indicates that the theoretical binding affinity, that can be cheaply estimated by molecular dynamics, can be safely used to select the best candidate peptides prior to experimental screening. This is at the basis of the success of the combined experimental and theoretical procedure described in this work.

\section{Selectivity of the peptides}

We have also considered the selectivity of the peptides toward CPT-11. Specifically, we carried out molecular dynamics (MD) simulation and experiments to analyze the stability of the SN-38-peptide complexes, being SN-38 the active metabolite of CPT-11 lacking the carbamate and the piperidylpiperidine groups (see Scheme 1). Results in Figure 4 show that peptide 1 is not selective for CPT-11 since it can also bind SN-38: the average value of the binding affinity over all the MD trajectory is approximately $-10 \mathrm{Kcal} / \mathrm{mol}$ and $-9 \mathrm{Kcal} / \mathrm{mol}$ for CPT-11 and SN-38 complex, respectively (Figure $4 \mathrm{~A})$.

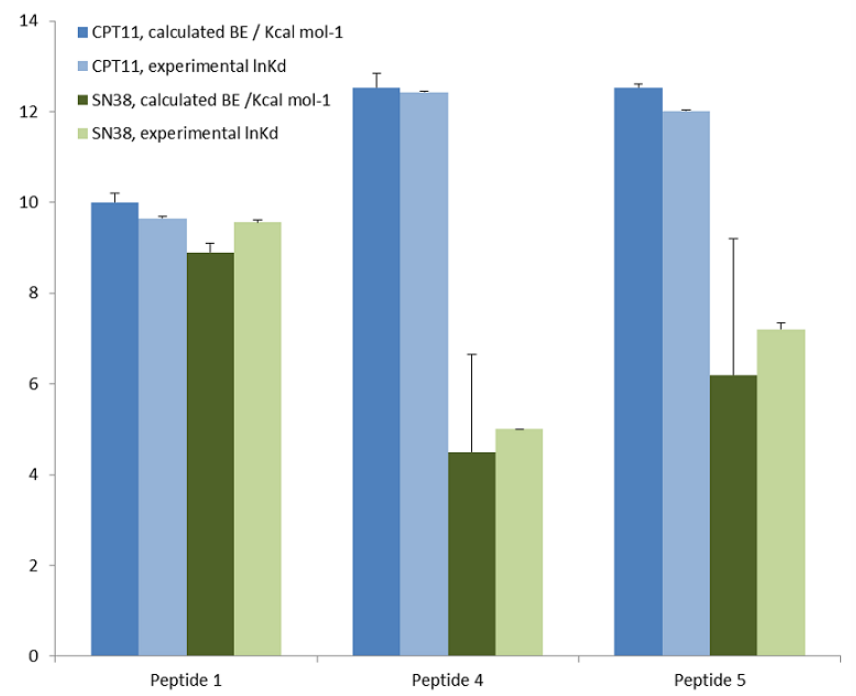

a

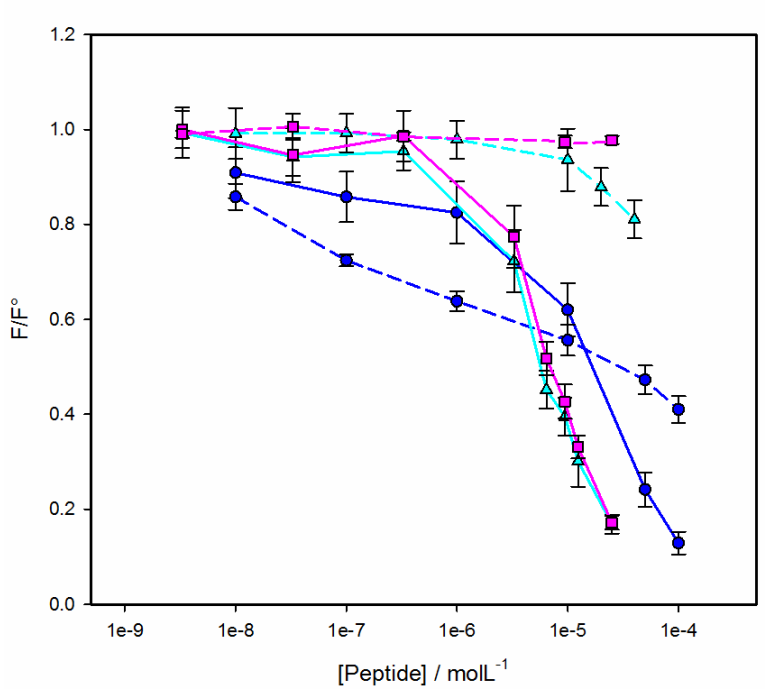

b

Figure 4

These results agree with the fluorescence binding experiment (Figure 4B), that also provide similar value for the binding affinity towards these two targets. On the other hand, MD simulations and experiments consistently show that peptides $\mathbf{4}$ and $\mathbf{5}$ are fully selective toward CPT-11 and unable to 
bind SN-38. Indeed, we have observed a dissociation of the complexes of SN-38 and peptides 4 and 5 along the MD trajectory (see Figure S3 in supplementary materials) and a very scarce fluorescence quenching in the corresponding experimental titrations (Figure 4B). The selectivity of peptides $\mathbf{4}$ and $\mathbf{5}$ toward CPT-11 further highlight the crucial role of the solvent environment in the stabilization of the peptide-drug complex and, thus, the need of design the peptides in an explicit solvent environment.

Remarkably, peptide $\mathbf{5}$ is extremely selective toward CPT-11 with a micromolar affinity while not binding at all SN-38. We further investigated the molecular basis of this striking difference in binding affinity since CPT-11 and SN-38 have rather similar structures (see Scheme 1). We gathered the atoms in CPT-11 in groups as shown in the Figure 1, calculating the number of contacts and the minimum distance between each group and the peptide. The results of this analysis show that the number of contacts between the piperidinyl-piperidine group and the carbamate one are significant. Moreover, the oxygen atom of this group remains at about $0.2 \mathrm{~nm}$ from the peptide during the whole MD trajectory (see Figure S4 and S5 in Supplementary Materials). Since this group is present only in the CPT-11, this could explain the selectivity of the peptide towards this molecule. These results show that the binding can be significantly altered, even by removing a single relevant moiety in the target, suggesting that the proposed procedure can design peptides capable of distinguish even targets that are chemically similar. We have also carried out a virtual alanine scanning on peptides 4 and 5 in order to identify, from the peptide side, the role of each amino acid in selectivity. Ten peptides were generated from peptide 4 and from peptide 5 by replacing each amino acid with alanine (the two cysteines were kept in order to maintain the disulphide bond), and we have evaluated the change in the VINA binding energy for each mutated peptide with respect with the original ones. The results are fully reported in Supplementary Materials (Figure S7). In peptide 4, the most relevant difference in binding of CPT-11 and SN38 is observed upon replacement of Tyr 3, Gln 4 and Tyr 8, which appear important in driving the selectivity of peptide 4 towards CPT-11. In peptide 5, the same role is played by Trp 2, Tyr 3 and $\operatorname{Trp} 4$. 


\section{Conclusion}

In this work we described the experimental validation of short peptides designed in silico as artificial receptors for the anticancer drug (CPT-11). The design procedure is tailored to produce peptides capable of recognizing their target in a specific environment, a denaturant in the case of terapeutic drug monitoring of CPT-11. Surface plasmon resonance and fluorescence spectroscopy confirms the theoretical predicted binding energies. Moreover, the designed peptides show a high selectivity towards CPT-11 respect to its very similar metabolite SN-38.

To the best of our knowledge, this is the first example of peptides designed in silico to bind their target in an organic solvent (i.e., methanol) whose activity is then proven experimentally. These results further demonstrate the power of our computational and experimental approach, showing evidence that short peptides designed in silico can be used as sensing elements for TDM of anticancer drugs. This discloses a convenient way to the development of unprecedented biosensors with biomolecules as sensing elements working in any organic solvent or at extreme conditions of temperature, pressure and $\mathrm{pH}$.

\section{Acknowledgements}

We acknowledge the AIRC 5x1000 grant "Application of Advanced Nanotechnology in the Development of Innovative Cancer Diagnostics Tools"

\section{References}

Alnaim, L., 2007. J. Oncol. Pharm. Pract. 13, 207-221.

Del Carlo, M., Capoferri, D., Gladich, I., Guida, F., Forzato, C., Navarini, L., Compagnone, D., Laio, A., Berti, F., 2016. ACS Sensors, 279-286.

De Jong, M.E., Huitema, A.D.R., Schellens, J.H.M., Rodenhuis, S., Beijnen, J.H. 2005. Clin. Pharmacokinet. 44, 147-173. 
Dey, J., Warner, I. M., 1997. J. Lumin. 71, 105-114.

Fassberg, J., Stella, V. J., 1992. J. Pharm. Sci. 81, 676 - 684.

Ferguson, B. S., Hoggarth, D.A., Maliniak, D., Ploense, K., White, R. J., Woodward, N., Hsieh, K., Bonham, A. J., Eisenstein, M., Kippin, T. E., Plaxco, K. W., Soh, H. T., 2014. Science Transl. Med. 5,213 ra165.

Gao, B., Yeap, S., Clements, A., Balakrishnar, B., Wong, M., Gurney, H., 2012. J. Clin. Oncol. 30, 4017-4025.

Gladich, I., Rodriguez, A., Hong Enriquez, R.P., Guida, F., Berti, F., Laio, A., 2015. J. Phys. Chem. B. $119,12963-12969$.

Hess, B., Kutzner, C., Van Der Spoel, D., \& Lindahl, E., 2008. Journal of chemical theory and computation, 4(3), 435-447.

Hong Enriquez, R. P., Pavan, S., Benedetti, F., Tossi, A., Savoini, A., Berti, F., Laio, A., $2012 . \quad$ J. Chem. Theor. Comput. 8, 1121-1128.

Hornak, V., Abel, R., Okur, A., Strockbine, B., Roitberg, A., \& Simmerling, C., 2006. Proteins, 65, $712-725$.

Ma, Q., Lu, A.Y.H., 2011. Pharmacol. Rev. 63, 437-459.

Marangon, E., Posocco, B., Mazzega, E., Toffoli, G.,2014. PLoS ONE 10, e0118194.

Paci, A., Veal, G., Bardin, C., Levêque, D., Widmer, N., Beijnen, J., Astier, A., Chatelut, E., 2014. Eur. J. Canc. 50, 2010-2019.

Trott, O., Olson, A.J., 2010. Comput. Chem., 31, 455-461.

Van Erp, N. P., de Wit, D., Guchelaar, H.- J., Gelderblom, H., Hessing, T. J., Hartigh, J. D., $2014 . \quad$ J. Chromatogr. B. 937, 33-43.

Walko, C.M., McLeod, H.L., 2014. J. Clin. Oncol. 32, 2581-2586. 
Widmer, N., Bardin, C., Chatelut, E., Paci, A., Beijnen, J., Levêque, D., Veal, G., Astier, A., 2014. Eur.

J. Canc. 50, 2020-2036.

\section{Tables}

Table 1. Binding data for the peptides designed in vacuum. ${ }^{\S}$ : The KGGG chain at the amino termini of the peptides was added as a spacer to allow immobilization of the peptides on the SPR chip surfaces; ${ }^{\text {a }}$ : dissociation constants from Hill analysis of the fluorescence quenching data and from (SPR titration); ${ }^{\text {b: }}$ Hill number from the fluorescence quenching data. ${ }^{\mathrm{c}}$ : in water; ${ }^{\mathrm{d}}:$ in methanol.

\begin{tabular}{|c|c|c|c|c|c|}
\hline \multirow[t]{2}{*}{ Peptide } & \multirow[t]{2}{*}{ Sequence ${ }^{\S}$} & \multirow{2}{*}{$\begin{array}{l}\mathrm{K}_{\mathrm{sv}} \\
10^{4} \mathrm{Lmol}^{-1}\end{array}$} & \multirow{2}{*}{$\begin{array}{l}\mathrm{K}_{\mathrm{Q}} \\
10^{12} \mathrm{Lmol}^{-1} \mathrm{~s}^{-1}\end{array}$} & \multirow{2}{*}{$\begin{array}{l}\mathrm{K}_{\mathrm{D}}^{\mathrm{a}} \\
10^{-6} \mathrm{molL}^{-1}\end{array}$} & \multirow[t]{2}{*}{$\mathrm{N}^{\mathrm{b}}$} \\
\hline & & & & & \\
\hline \multirow[t]{2}{*}{1} & KGGGCVHYDMHQYWMC & $6.52 \pm 0.27^{c}$ & $18.5^{c}$ & $23.4 \pm 0.9^{c}\left(64 \pm 2^{c}\right)$ & $0.89^{c}$ \\
\hline & & $5.09 \pm 0.12^{\mathrm{d}}$ & $14.2^{\mathrm{d}}$ & $41.3 \pm 0.6^{\mathrm{d}}$ & $1.01^{\mathrm{d}}$ \\
\hline \multirow[t]{2}{*}{2} & KGGGCIRNRWNYQYRC & $4.50 \pm 0.17^{c}$ & $11^{d}$ & $20.2 \pm 0.9^{c}\left(63 \pm 3^{c}\right)$ & $0.79^{c}$ \\
\hline & & $1.90 \pm 0.05^{\mathrm{d}}$ & $5.1^{\mathrm{d}}$ & $53 \pm 2^{d}$ & $1.03^{\mathrm{d}}$ \\
\hline \multirow[t]{2}{*}{3} & KGGGCWPTGNYYNFGC & $3.8 \pm 0.08^{c}$ & $9.5^{c}$ & $26 \pm 1^{c}\left(62 \pm 2^{c}\right)$ & $0.81^{c}$ \\
\hline & & $1.7 \pm 0.06^{\mathrm{d}}$ & $4.6^{\mathrm{d}}$ & $56 \pm 2^{\mathrm{d}}$ & $1.04^{\mathrm{d}}$ \\
\hline
\end{tabular}

Table 2. Binding data for the peptides designed in methanol. ${ }^{\mathrm{a}}$ : in methanol; ${ }^{\mathrm{b}}$ : dissociation constants from Hill analysis of the fluorescence quenching data; ${ }^{c}$ : Hill number from the fluorescence quenching data.

\begin{tabular}{|c|c|c|c|c|c|}
\hline \multirow[t]{2}{*}{ Peptide } & \multirow[t]{2}{*}{ Sequence } & $\mathrm{K}_{\mathrm{sv}}{ }^{\mathrm{a}}$ & $\mathrm{K}_{\mathrm{Q}}^{\mathrm{a}}$ & $\mathrm{K}_{\mathrm{D}}^{\mathrm{a}, \mathrm{b}}$ & $\mathrm{N}^{\mathrm{a}, \mathrm{c}}$ \\
\hline & & $10^{4} \mathrm{Lmol}^{-1}$ & $10^{12} \mathrm{Lmol}^{-1} \mathrm{~s}^{-1}$ & $10^{-6} \mathrm{molL}^{-1}$ & \\
\hline 4 & KGGGCQYQYEIYYYYC & $112 \pm 8$ & 314 & $4.1 \pm 0.13$ & 0.99 \\
\hline
\end{tabular}




\section{Figure Legends.}

Figure 1. a) the outcome for a typical design alghoritm run, starting from a poly-valine peptide: the mean values of Vina score calculated on the design trajectories is given as a function of mutation number. The different colors correspond to different efficacious temperatures (see the text). b): complex peptide-CPT-11; the complex results to be stable with the CPT-11 (orange) inside the peptide ring.

Figure 2. Binding of CPT-11 by the peptides designed in vacuum. a-c: SPR sensorgrams and their corresponding binding isotherms, obtained immobilizing peptides $\mathbf{1}$ (blue); $\mathbf{2}$ (red) and $\mathbf{3}$ (green) on the sensor chip and increasing CPT-11 concentration. d: Decay of fluorescence emission of $1 \mu \mathrm{M}$ CPT-11 at $430 \mathrm{~nm}$ upon addition of increasing concentrations of peptides.

Figure 3. Binding of CPT-11 in methanol. a) Decay of fluorescence emission of $0.5 \mu \mathrm{M} \mathrm{CPT-11}$ at 430 $\mathrm{nm}$ upon addition of increasing concentrations of peptides in methanol. b) Experimental affinity to CPT11 for peptides 1 to $\mathbf{5}$ vs. the predicted binding energy calculated by molecular dynamics. Theoretical and experimental results are truly compatible, and give the same hierarchy of the binding affinity for all the considered peptides. The shift between the two groups consisting by peptides $\mathbf{1}, \mathbf{2}$ and $\mathbf{3}$ and peptides $\mathbf{4}$ and $\mathbf{5}$ respectively, clearly shows the algorithm capability of designing environment-specific peptides.

Figure 4. Selectivity of the peptides towards CPT-11 and SN-38. a) experimental and predicted affinities to CPT-11 and SN-38 for peptides 1, 4 and 5. b) fluorescence emission decay of $1 \mu \mathrm{M}$ CPT-11 (solid lines) and SN-38 (dotted lines) upon addition of increasing concentrations of peptide $\mathbf{1}$ (blue), 4 (pink) and $\mathbf{5}$ (cyan) in methanol. 
